CERN-TH/95-286

ROME1-1121/95

hep-ph/9511259

\title{
Light Higgsino Detection at LEP1.5
}

\author{
S. Ambrosanio*, B. Mele \\ Dipartimento di Fisica, Università "La Sapienza" and I.N.F.N., Sezione di Roma, \\ P.le Aldo Moro 2, I-00185 Rome, Italy
}

M. Carena, C.E.M. Wagner

CERN, TH Division, CH-1211 Geneva 23, Switzerland

\begin{abstract}
Within the minimal supersymmetric extension of the Standard Model, the best fit to the most recent precision-measurement data requires charginos and neutralinos, with dominant Higgsino components and with masses within the reach of LEP1.5 $(\sqrt{s}=140 \mathrm{GeV})$. In this work, we present a detailed analysis of the neutralino and chargino production processes for the favoured region of parameter space, that is low values of $|\mu|$ and either low or large values of $\tan \beta$. We find that chargino and neutralino searches can cover the Higgsino region in the $\left(\mu, M_{2}\right)$ plane for values of $M_{2} \lesssim 1 \mathrm{TeV}$, at the next phases of the LEP collider. We also show that, due mainly to phase-space constraints, the lightest neutralinos should be more easily detectable than charginos in most of the parameter space preferred by precision-measurement data.
\end{abstract}

CERN-TH/95-286

October 1995

* Address after December 1, 1995: University of Michigan, 3068A H.M. Randall Physics Lab., 500 East University, Ann Arbor, MI 48109-1120, U.S.A. 

The minimal supersymmetric extension of the Standard Model allows a solution of the gauge hierarchy problem and can be obtained as a low energy effective theory of supersymmetric grand unified theories including also gravity [1]. The model contains a large number of free parameters associated with the soft breakdown of supersymmetry, which lead to large uncertainties in the supersymmetric particle discovery potential of present and future colliders [2]. In particular, the physical properties of the chargino and neutralino sectors of the theory depend on four unknown parameters [3]: the soft supersymmetry breaking mass parameters of the supersymmetric partners of the $S U(2)_{L}$ and $U(1)_{Y}$ gauge bosons, which we shall denote by $M_{2}$ and $M_{1}$, respectively, the Higgs supersymmetric mass parameter $\mu$, and the ratio of Higgs vacuum expectation values $\tan \beta$. If the theory proceeds from a grand unified theory at very high energies, which will be implicitly assumed within this work, the low energy values of the gaugino masses are related by $M_{1} \simeq M_{2} \alpha_{1} / \alpha_{2}$, reducing to three the number of relevant free parameters.

The existence of light supersymmetric particles in nature can be tested through direct experimental detection, and also through deviations of the precision measurement data from the Standard Model predictions. Supersymmetric particles can, indeed, affect the low-energy observables through loop radiative corrections, which become negligibly small as soon as the supersymmetric particle masses are far above the electroweak scale. The most recent precision-measurement data show that the ratio of the width of $Z$ to bottom quarks to its total hadronic decay width, $R_{b}=\Gamma_{b} / \Gamma_{h}$, is more than three standard deviations above the Standard Model prediction for this quantity [4]. This deviation can be partially explained by the presence of supersymmetric particles at the weak scale. The best fit to the precision-measurement data leads to strong constraints on two of the three independent parameters of the neutralino and chargino sector of the theory: $\mu$ and $\tan \beta$ [5]-11].

Within the MSSM, large one-loop corrections to $R_{b}$ are always associated with large values of the third-generation Yukawa couplings. The supersymmetric top and bottom Yukawa couplings are related to their running mass values by:

$$
h_{t}=\frac{m_{t}}{\sin \beta}, \quad h_{b}=\frac{m_{b}}{\cos \beta} .
$$

From Eq. (四), it is clear that in order to enhance the top (bottom) quark Yukawa coupling effects, $\tan \beta$ should acquire the smallest (largest) value allowed by the theory [7, 10]. The requirement that the Yukawa couplings remain in the perturbative domain up to scales of the order of the grand unification scale $M_{G U T} \simeq 10^{16} \mathrm{GeV}$ implies that the largest corrections are obtained either at values of $\tan \beta \simeq m_{t} / m_{b}$ [9] or at the infrared fixed-point solution for the top quark mass [8]. Interestingly enough, for the values of the top quark mass measured at the Tevatron collider [12], these regions of the parameter space are also preferred [13] from the point of view of Yukawa coupling unification at a scale $M_{G U T}$ [14, 15.

In the low $\tan \beta$ regime, large positive corrections to $R_{b}$ may only be obtained through the one-loop chargino-stop contributions [5], which are enhanced for light stops, predominantly right-handed, and for chargino masses close to $M_{Z} / 2$. These effects are most relevant for low $|\mu|$ values, i.e. $|\mu| \lesssim M_{Z}$, for which the Higgsino component of the lightest chargino is enhanced and the chargino-stop-bottom coupling is 
approximately given by $h_{t}$, Eq. (四). For large values of $\tan \beta$, large positive corrections may also be obtained from both the neutral Higgs sector of the theory and from neutralino-sbottom loops. Similarly to the low $\tan \beta$ case, the genuine supersymmetric contributions are maximized for low values of the supersymmetric mass parameter $|\mu| \lesssim M_{Z}$. There are also strong restrictions on the lightest stop and sbottom particles, which should be light and predominantly right-handed to avoid unacceptable corrections to the $W^{ \pm}$mass or to the $Z$ leptonic width, but do not play a direct role in the chargino and neutralino production processes.

In this paper, we study the potential of LEP1.5 (the LEP phase at $\sqrt{s} \simeq 140 \mathrm{GeV}$ that started running at the end of October 1995) to explore the range of parameters suggested by precision measurements through neutralino and chargino searches. The phase 1.5 of the LEP collider is expected to collect an integrated luminosity of 5$10 \mathrm{pb}^{-1}$. We also extend our analysis to the higher-energy $(\sqrt{s} \simeq 190 \mathrm{GeV})$ operation phase of LEP2.

Neutralino production at LEP can proceed through the process [16]-18]:

$$
e^{+} e^{-} \rightarrow \widetilde{\chi}_{1}^{0} \widetilde{\chi}_{2}^{0}
$$

where $\tilde{\chi}_{1}^{0}$ and $\tilde{\chi}_{2}^{0}$ are the lightest and next-to-lightest neutralino particles, respectively. This leads to a very interesting signal, since the $\tilde{\chi}_{1}^{0}$ escapes detection and the $\tilde{\chi}_{2}^{0}$ decay products are hence completely unbalanced in energy and momentum. Pair production of the lightest neutralino contributes to the $Z$-boson invisible decay width and is hence not relevant for neutralino detection at energies above the $Z$ pole.

Light charginos are mainly produced in pairs [17, 20]:

$$
e^{+} e^{-} \rightarrow \tilde{\chi}_{1}^{+} \tilde{\chi}_{1}^{-}
$$

and, due to their short lifetime, can be searched for through their three-body decays into the lightest neutralino plus a light fermion pair.

For particular parameter configurations, the production rate of light charginos and neutralinos at LEP also suffers from large uncertainties related to the neutral and charged slepton spectrum, respectively. The uncertainties are larger when the gaugino components of the neutralinos/charginos are more important. Indeed, due to the smallness of the electron Yukawa coupling, the strength of the slepton coupling to electrons is just a reflection of the largeness of the gaugino components in charginos and neutralinos. On the other hand, if the light charginos and neutralinos are predominantly Higgsinos, as suggested by precision measurements, the main production mechanism is through $s$-channel $Z / \gamma$ exchange and the production cross sections may be accurately determined. Requiring chargino masses smaller than $M_{Z}$, Higgsino dominance occurs for values of $|\mu| \lesssim M_{Z}$. In this work, we will concentrate on the range $2 M_{Z}<M_{2} \lesssim 1 \mathrm{TeV}$ and $|\mu| \lesssim M_{Z}$, in which the ratio $M_{2} /|\mu|$ guarantees a dominant Higgsino component in light neutralinos/charginos and the values of $M_{2}$ can still be considered "natural".

As we will show, the production rates are large for both neutralinos and charginos in the above scenario. On the other hand, when $M_{2}$ is increased, $\widetilde{\chi}_{1}^{+}$and $\widetilde{\chi}_{1}^{0}$ tend to be degenerate in mass. The same holds, but to a lesser extent, for $\widetilde{\chi}_{2}^{0}$ and $\tilde{\chi}_{1}^{0}$. Indeed, the 
relation $m_{\widetilde{\chi}_{2}^{0}}>m_{\widetilde{\chi}_{1}^{ \pm}}>m_{\widetilde{\chi}_{1}^{0}}$ holds in all the regions of the plane $\left(\mu, M_{2}\right)$ considered here (apart from a small region already excluded by LEP1 data). The actual production of $\widetilde{\chi}_{1}^{0} \widetilde{\chi}_{2}^{0}$ and $\tilde{\chi}_{1}^{+} \tilde{\chi}_{1}^{-}$pairs is observed through the decays $\emptyset$ :

$$
\begin{aligned}
& \left.\tilde{\chi}_{2}^{0} \rightarrow \tilde{\chi}_{1}^{0}+\text { (visible }\right) \\
& \tilde{\chi}_{1}^{ \pm} \rightarrow \tilde{\chi}_{1}^{0}+(\text { visible }) .
\end{aligned}
$$

Since $\widetilde{\chi}_{2}^{0}\left(\widetilde{\chi}_{1}^{ \pm}\right)$is produced with rather low velocity, if the mass splitting between the next-to-lightest neutralino (chargino) and the lightest neutralino is small, the decay products may not have sufficient energy and multiplicity to pass the normal experimental trigger for missing energy and momentum events. Indeed, in the Higgsino region, these mass splittings are naturally small, of order $M_{W}^{2} / M_{2}$. Presently, in Monte Carlo simulations that study supersymmetric signals and backgrounds at LEP [21], a mass splitting:

$$
\begin{aligned}
\Delta_{ \pm} & \equiv m_{\widetilde{\chi}_{1}^{ \pm}}-m_{\widetilde{\chi}_{1}^{0}} \gtrsim 10 \mathrm{GeV} \\
\Delta_{0} & \equiv m_{\widetilde{\chi}_{2}^{0}}-m_{\widetilde{\chi}_{1}^{0}} \gtrsim 10 \mathrm{GeV}
\end{aligned}
$$

is required for the events to be observable. In the following, we prove that, after imposing cuts on the mass splittings as in Eqs. (6) and (77), neutralino searches are more efficient than chargino searches in covering the $\left(\mu, M_{2}\right)$ Higgsino region.

Cross sections for the processes Eqs.(2) and (3) at the LEP1.5 energy are shown in Figs. 1 and 2, at low values (close to the fixed point value for $M_{t}=170 \mathrm{GeV}$ ) and large values of $\tan \beta$, as preferred by precision measurements, in the plane $\left(\mu, M_{2}\right)$. In particular, we have fixed $\tan \beta=1.2$ and 50, respectively. The effect of QED initial state radiation is included. All the slepton masses are set at $300 \mathrm{GeV}$. However, we checked that varying slepton masses between $50 \mathrm{GeV}$ and $1 \mathrm{TeV}$ does not cause any noticeable change in the cross sections, in the range of $\left(\mu, M_{2}\right)$ considered here. We have also checked that the low $\tan \beta$ results are basically unchanged for $\tan \beta$ values that are closer to (or somewhat further away from) 1.

The LEP1 and LEP1.5 kinematical limits are shown in the figures. One can observe that, in almost all the region kinematically covered by LEP1.5, cross sections are as large as several picobarns for both low and large values of $\tan \beta$. For an integrated luminosity of $10 \mathrm{pb}^{-1}$, one expects up to more than 100 events from neutralino (chargino) production, before experimental cuts are applied.

In all the figures, dashed lines give contours for the splitting of the decaying particle mass and the lightest neutralino mass, $\Delta_{0}$ and $\Delta_{ \pm}$. One can see that, in general, imposing the conditions in Eqs. (6) and (7) excludes a much larger portion of the parameter space in the chargino case, for both low and high $\tan \beta$ values. On the other hand, for neutralinos the phase-space restriction in Eq. (7) is met up to very large values of $M_{2}$, of order $1 \mathrm{TeV}$. While chargino cross sections are comparable to the neutralino ones, $\widetilde{\chi}_{1}^{ \pm}$is always considerably closer in mass to $\widetilde{\chi}_{1}^{0}$ than $\widetilde{\chi}_{2}^{0}$, so that chargino detection becomes more difficult at high $M_{2}$, mostly due to phase-space constraints. In particular, Eq. (6) is fulfilled only for $M_{2} \lesssim(5-6) M_{Z}$ in both the low and high

\footnotetext{
${ }^{1}$ Additional missing energy, in the form of neutrinos, may be present
} 
$\tan \beta$ regime. Moreover, the larger unbalance in energy and momentum in the case of neutralino final states further enhances the advantages of the $\widetilde{\chi}_{1}^{0} \widetilde{\chi}_{2}^{0}$ channel.

Hence, if light neutralinos with a mass below the kinematic limit and a dominant Higgsino component are present in the theory, they should be more easily detected, at the next phase of the LEP collider. Possible invisible decays of the next-to-lightest neutralino and cascade decays through a chargino (that are even more constrained by phase space) can deplete the observable neutralino production rate. On the other hand, one can see that, for Higgsino dominated compositions, the $\widetilde{\chi}_{2}^{0}$ decays into neutrinos with a BR less than $20 \%$ and the cascade-decay fraction can reach at most $20-30 \%$ 19. Hence, most of the $\widetilde{\chi}_{2}^{0}$ decays should have three body visible final states.

We stress that the mass splitting we are requiring for neutralino (chargino) detection, Eqs. (6) and (7), is based on the present experimental analysis [22], which shows that neutralino (chargino) searches for mass splittings $5 \mathrm{GeV}<\Delta_{0}\left(\Delta_{ \pm}\right)<10 \mathrm{GeV}$ are remarkably more difficult and require a dedicated experimental trigger on the energy and multiplicity of the events. More extreme cases with $\Delta_{0}\left(\Delta_{ \pm}\right)<5 \mathrm{GeV}$ seem, of course, even more challenging. It is clear, however, that a definite statement on the neutralino and chargino observability requires a detailed study of the signal versus possible backgrounds through Monte Carlo simulations that can mimic the effect of experimental triggers and kinematical cuts.

We have also considered Higgsino production at LEP2 (Figs. 3 and 4). The last phase of the LEP collider will reach an energy of about $190 \mathrm{GeV}$ and a total luminosity of $300 \mathrm{pb}^{-1}$. In this case, although the total cross section goes down by a factor 3 or 4 , the luminosity is sufficiently high to discriminate the presence of light supersymmetric particles in a clear way. However, the production processes at these energies suffer from a large background from $W^{+} W^{-}$and $Z Z$ production in both the hadronic and leptonic modes, which is suppressed at the LEP1.5 center of mass energies. One can see in Figs. 3 and 4 that the outcome at LEP2 is analogous to the one at LEP1.5. The high- $M_{2}$ region is more challenging for chargino than for neutralino searches. Nevertheless, despite the higher luminosity, the background analysis could be more involved at larger $\sqrt{s}$.

In conclusion, we have shown that there is a clear indication that neutralino searches are at least as competitive as chargino ones, to explore the Higgsino region in the $\left(\mu, M_{2}\right)$ plane. In particular, neutralino searches can cover this region of parameters up to almost the kinematical limit, for values of $\tan \beta$ favoured by precision measurements and values of $M_{2} \lesssim 1 \mathrm{TeV}$. In the case of charginos, the kinematic reach in the $\left(\mu, M_{2}\right)$ plane is almost equivalent. On the other hand, the phase-space constraints in chargino decays make its detection more challenging than in neutralino production processes. A similar result is valid also for intermediate $\tan \beta$ values.

Acknowledgements: We would like to thank P. Chankowski, C. Dionisi, M. Felcini and J. F. Grivaz for useful comments and discussions. Research supported in part by the European Union under contract No. ERB-CHRX-CT93-0132. 


\section{References}

[1] S. Dimopoulos, S. Raby and F. Wilczek, Phys. Rev. D24 (1981) 1681;

S. Dimopoulos and H. Georgi, Nucl. Phys. B193 (1981) 150;

L. Ibañez and G. G. Ross, Phys. Lett. 105B (1981) 150;

H.P. Nilles, Phys. Rep. 110 (1984) 1.

[2] H. E. Haber and G. L. Kane, Phys. Rep. 117 (1985) 75.

[3] J. Ellis and G. G. Ross, Phys. Lett. 117B (1982) 397;

J. M. Frère and G. L. Kane, Nucl. Phys. B223 (1983) 331;

J. F. Gunion and H. E. Haber, Nucl. Phys. B272 (1986) 1; B402 (1993) 567;

A. Bartl, H. Fraas, W. Majerotto and N. Oshimo, Phys. Rev. D40 (1989) 1594.

[4] P. Antilogus et al. [LEP Electroweak Working Group], LEPEWWG/95-02 (1995).

[5] M. Boulware and D. Finnel, Phys. Rev. D44 (1991) 2054.

[6] G. Altarelli, R. Barbieri and F. Caravaglios, Phys. Lett. 314B (1993) 357.

[7] J. D. Wells, C. Kolda and G. L. Kane, Phys. Lett. 338B (1994) 219.

[8] M. Carena and C. E. M. Wagner, Nucl. Phys. B452 (1995) 45.

[9] D. Garcia and J. Sola, Phys. Lett. 354B (1995) 335.

[10] P. Chankowski and S. Pokorski, to appear in Proc. Beyond the Standard Model IV, Lake Tahoe, CA, December 1994; MPI Preprint MPI-PhT/95-49.

[11] A. Dabelstein, W. Hollik and W. Mösle, Univ. of Karlsruhe Preprint KA-THEP-5-1995.

[12] F. Abe et al. [CDF Collaboration], Phys. Rev. Lett. 74 (1995) 2626;

S. Abachi et al. [D0 Collaboration], Phys. Rev. Lett. 74 (1995) 2632.

[13] C. E. M. Wagner, talk presented at the Conference SUSY95, Palaiseau, France, June 1995, to appear in the Proceedings, CERN Preprint CERN-TH/95-261, October 1995, hep-ph/9510341.

[14] H. Arason et al., Phys. Rev. Lett. 67 (1991) 2933;

V. Barger, M. S. Berger and P. Ohmann, Phys. Rev. Lett. 49 (1994) 4908;

P. Langacker and N. Polonsky, Phys. Rev. D47 (1993) 4028; D49 (1994) 1454;

M. Carena, S. Pokorski and C. E. M. Wagner, Nucl. Phys. B406 (1993) 59;

W. A. Bardeen, M. Carena, S. Pokorski and C. E. M. Wagner, Phys. Lett. 320B (1994) 110.

[15] M. Olechowski and S. Pokorski, Phys. Lett. 214B (1988) 393;

B. Anantharayan, G. Lazarides and Q. Shafi, Phys. Rev. D44 (1991) 1613;

S. Dimopoulos, L. J. Hall and S. Raby, Phys. Rev. Lett. 68 (1992) 1984, Phys. Rev. D45 (1992) 4192. 
[16] A. Bartl, H. Fraas and W. Majerotto, Nucl. Phys. B278 (1986) 1.

[17] D. A. Dicus and X. Tata, Phys. Rev. D35 (1987) 2110;

M. Chen, C. Dionisi, M. Martinez and X. Tata, Phys. Rep. 159 (1988) 201;

J. F. Grivaz et al., in Proc. of the ECFA Workshop on LEP200, Aachen, Sept. 29-Oct. 1, 1986, eds. A. Böhm and W. Hoogland, CERN 87-08, ECFA 87/108, Geneva, (1987) Vol. II, p. 380.

[18] S. Ambrosanio and B. Mele, Phys. Rev. D52 (1995) 3900.

[19] S. Ambrosanio and B. Mele, "Neutralino Decays in the Minimal Supersymmetric Standard Model", Preprint ROME1-1095/95, August 1995, to be published in Physical Review D, hep-ph/9508237.

[20] A. Bartl, H. Fraas and W. Majerotto, Z. Phys. C30 (1986) 441;

A. Bartl, H. Fraas, W. Majerotto and B. Mösslacher, Z. Phys. C55 (1992) 257.

[21] "Interim Report on the Physics Motivations for an Energy Upgrade of LEP2", CERN Preprint CERN-TH/95-151, CERN-PPE/95-78.

[22] C. Dionisi, private communication. 


\section{Figure captions}

Fig. 1 Cross section and kinematics, in the $\left(\mu, M_{2}\right)$ plane, of the processes $e^{+} e^{-} \rightarrow \widetilde{\chi}_{1}^{0} \widetilde{\chi}_{2}^{0}$ (a) and $e^{+} e^{-} \rightarrow \tilde{\chi}_{1}^{+} \tilde{\chi}_{1}^{-}$(b) at LEP1.5, in the $\tan \beta=1.2$ case. Contour lines for cross sections (in pb) are represented by solid black lines. The grey lines represent the LEP1 and LEP1.5 kinematical reach for the process. The dashed lines and the bold labels in Fig. 1a (b) give isocontours in GeV for the quantity $\Delta_{0}$ $\left(\Delta_{ \pm}\right)$, defined in Eq. (可) (Eq. ([7))

Fig. 2 The same as in Fig. 1 , but in the $\tan \beta=50$ case.

Fig. 3 The same as in Fig. 1, but for LEP2 energies.

Fig. 4 The same as in Fig. 3, but in the $\tan \beta=50$ case. 


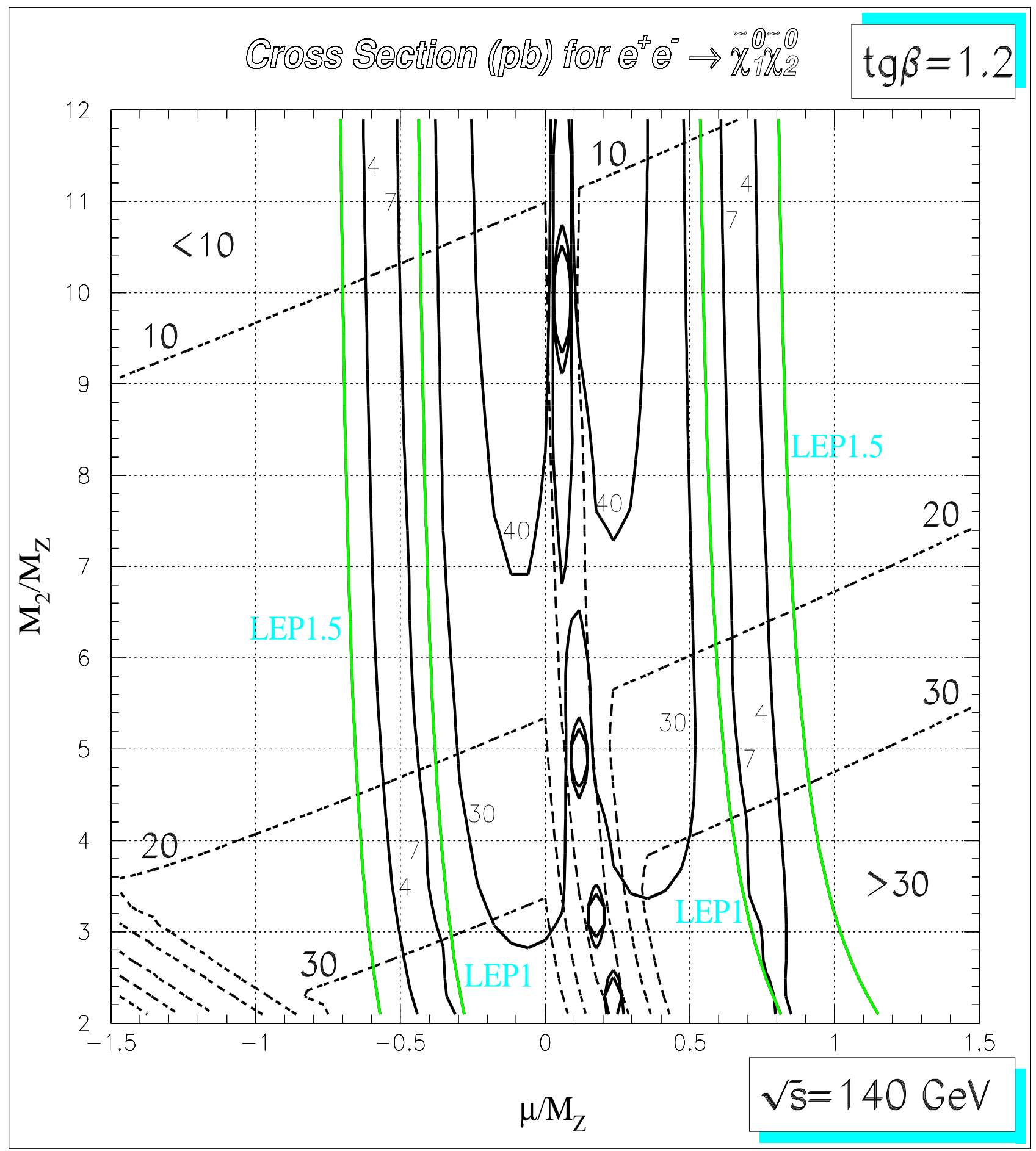

Figure 1a 


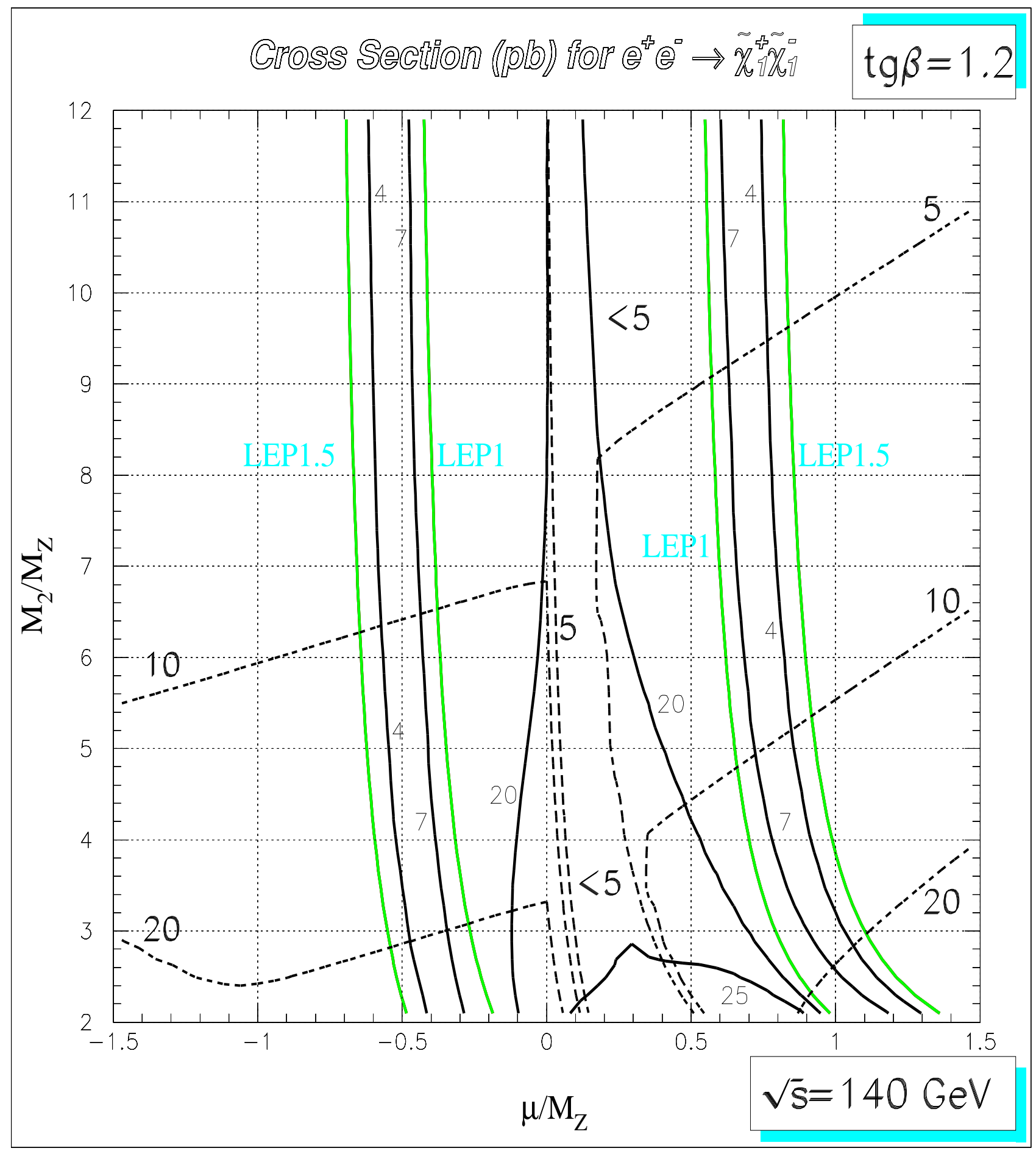

Figure 1b 


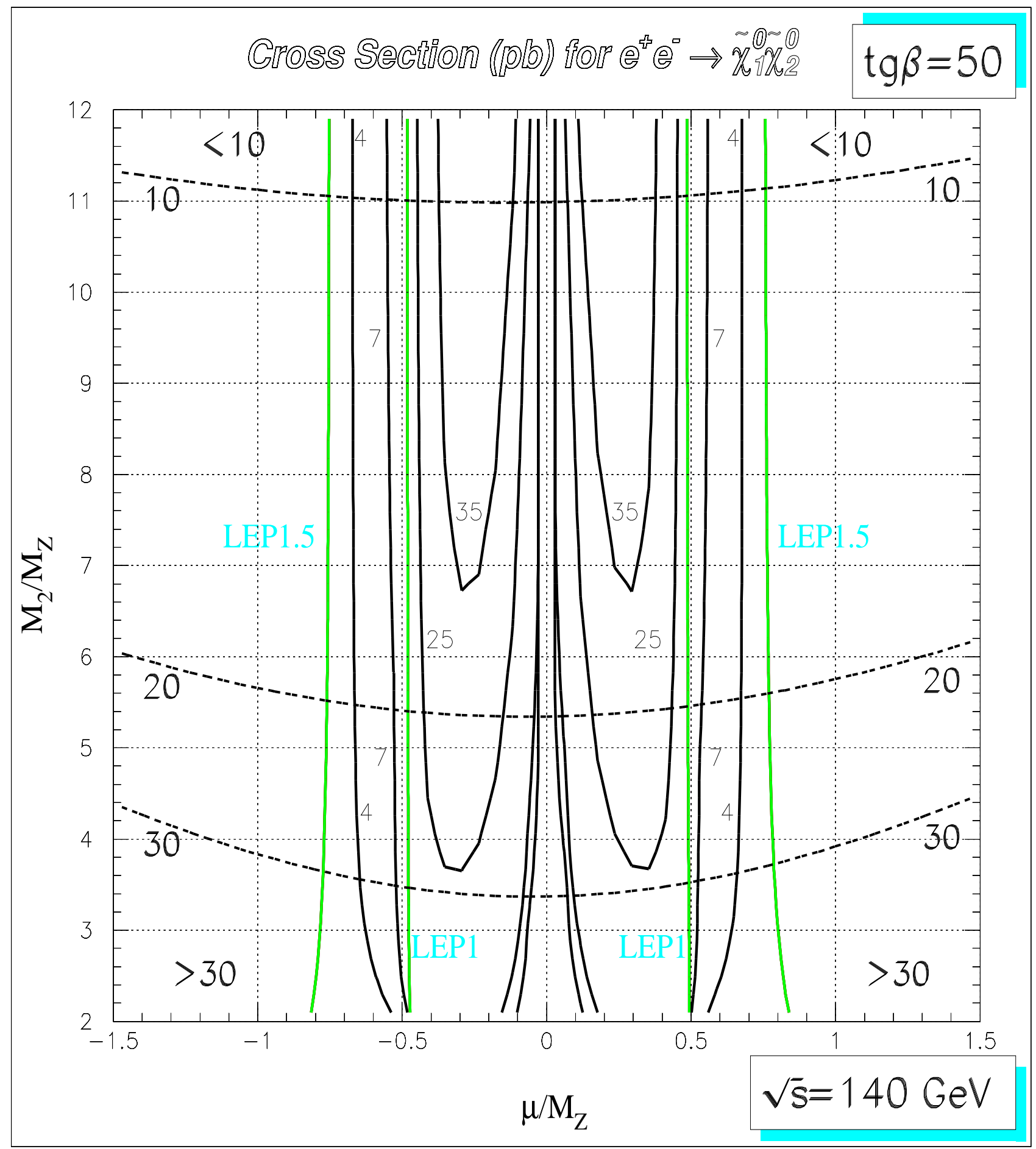

Figure 2a 


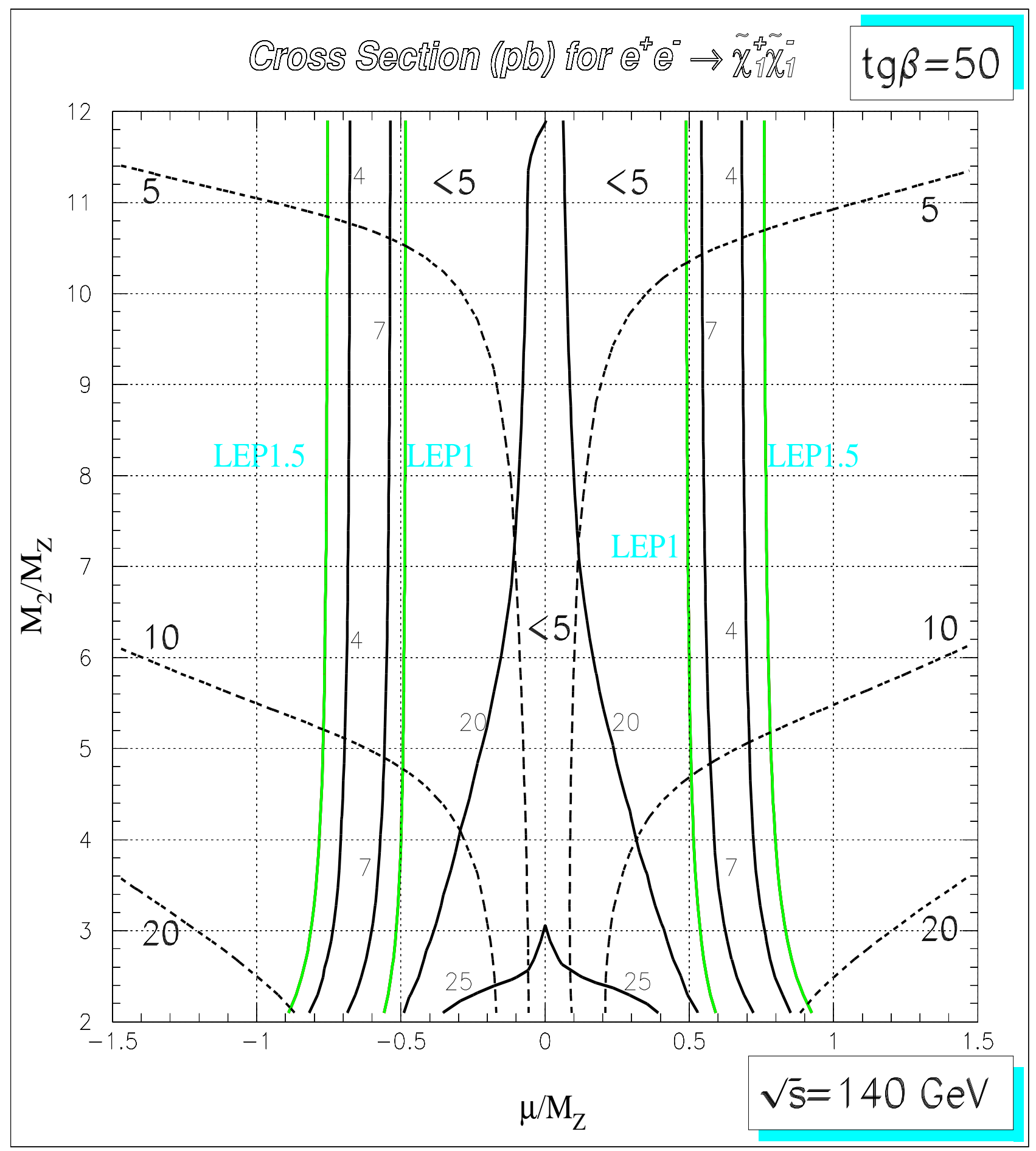

Figure 2b 


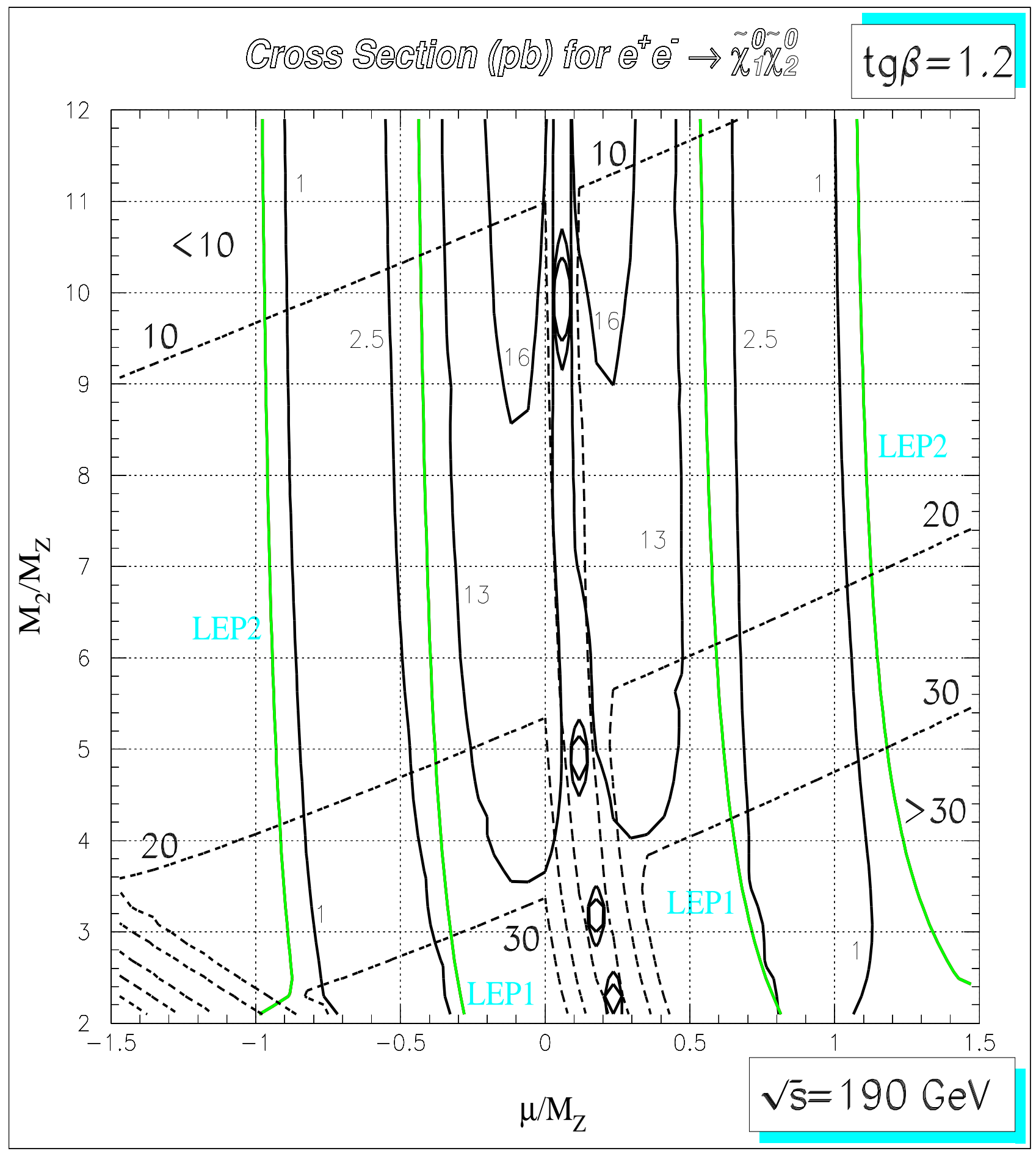

Figure 3a 


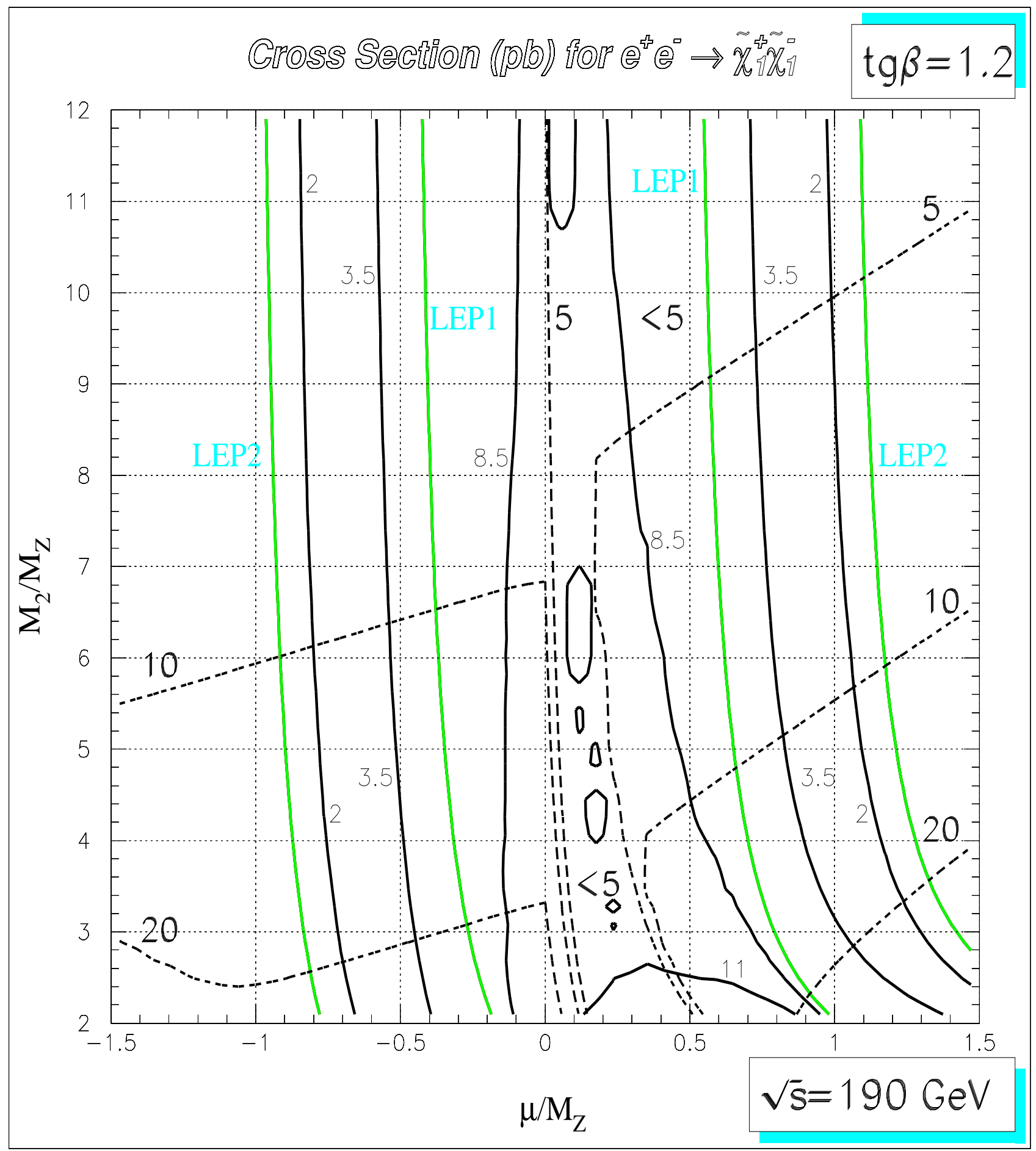

Figure 3b 


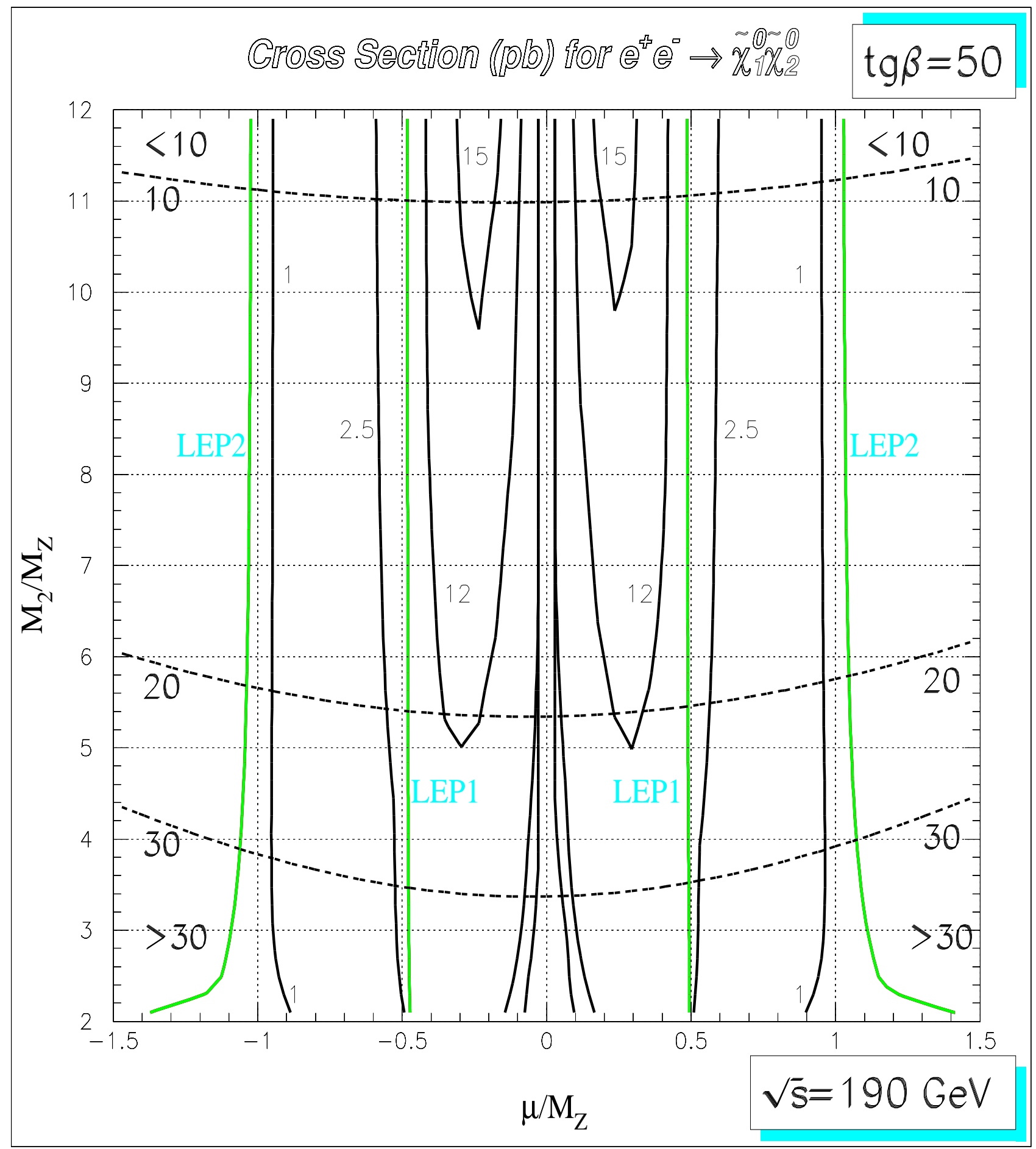

Figure 4a 


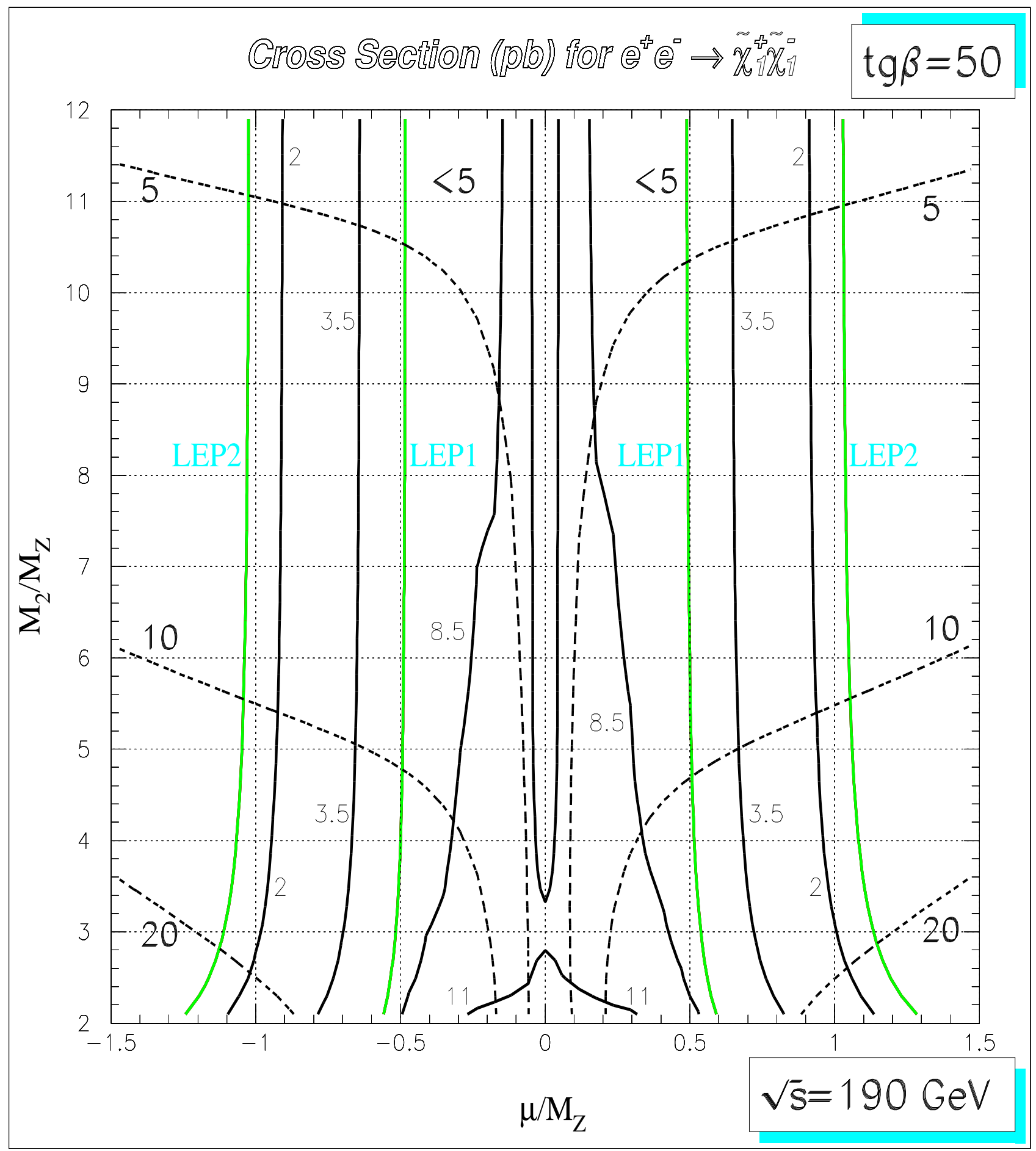

Figure 4b 